\title{
Clustering of Compressed Illumination-Invariant Chromaticity Signatures for Efficient Video Summarization
}

Mark S. Drew and James Au

School of Computing Science, Simon Fraser University,

Vancouver, B.C. Canada V5A 1S6

$\{$ mark,ksau\}@cs.sfu.ca

www.cs.sfu.ca/ mark 


\begin{abstract}
Motivated by colour constancy work in physics-based vision, we develop a new low-dimensional video frame feature that is effectively insensitive to lighting change and apply the feature to keyframe production using hierarchical clustering. The new image feature results from normalising colour channels for frames and then treating $2 \mathrm{D}$ histograms of chromaticity as images and compressing these. Because we effectively reduce any video to the same lighting conditions, we can precompute a universal basis on which to project video frame feature vectors. The new feature thus has the advantage of more expressively capturing essential colour information, and is useful for video indexing because it is very low-dimension - the feature vector is only of length 8 . We carry out clustering efficiently by adapting the hierarchical clustering data structure to temporally-ordered clusters. Using a new multi-stage hierarchical clustering method, we merge clusters based on the ratio of cluster variance to variance of the parent node, merging only adjacent clusters, and then follow with a second round of clustering. The second stage merges clusters incorrectly split in the first round by the greedy hierarchical algorithm, and as well merges non-adjacent clusters to fuse near-repeat shots. The new summarization method produces a very succinct set of keyframes for videos and, compared to a previous well-known technique, results are excellent.
\end{abstract}

Keywords: Colour, Chromaticity, Video Summarization, Clustering, Illumination Invariance

\title{
1 Introduction
}

Amongst expressions of video summarization, keyframes remain the most natural and immediate means of reducing video information to a concise form. As well, if we adopt keyframes as our goal then "inverse Hollywood" techniques, such as graph-based pruning [21] or other hierarchical representations of video sequences [22, 25, 16] can be brought to bear.

As well, if keyframes are established by means of extracting features from frames, then these same features can be used for indexing of video sequences. Here we are interested in generating feature vectors that are as small as possible for purposes of indexing while maintaining as much as possible the saliency of features used. For image database applications, we have found that quite a careful application of a succession of steps was necessary to generate the most meaningful, expressive features for images. For video, in fact the job is in a sense more difficult in that rather than differentiating between images of apples and oranges, as in image indexing, here we which to distinguish temporal changes of image sequences of similar frames and establish the most salient frame as the representative for each shot.

Since illumination change has been found to be key, triggered either by actual change in lighting by shadows, say, or simply by changes in shading due to viewpoint shifts, as a first step we approximately but effectively discount the effects of illumination change by normalising each colour band, R, G, and B, to length 1 . Then, after this normalization, we form 2D chromaticity histograms. Since such histograms can be rather sparse, and since we need smaller feature vectors, we go on to treat these 2D functions as images, and compress them as if they were images.

We have found that generating coarsely sampled histograms will simply not do - too much of the structure that characterises colour features is lost [8]. Instead, a wavelet-based compression was found to be worthwhile, since this method preserves local structure sufficiently such that the histogram intersection for objects under differing lighting is always higher than just using larger histogram bins.

However, we found that compression of histograms could be still further improved (and simplified) if the histograms are first binarised; i.e., entries are replaced with 1 or 0 . In effect, we are replacing histograms by a kind of colour signature, similar to a palette. This turns out to have the concomitant positive effect of producing many fewer negatives in the compressed histogram. 
We found that size $16 \times 16$ for compressed histograms gave good results. From such a histogram, we would like to pick up the salient features that contribute most to the "energy" in the histogram. In order to be able to fine tune performance by changing the number of parameters in small increments, instead of continuing to smaller histograms by further compression we perform a DCT on the $16 \times 16$ histograms. Using zigzag order for the coefficients, we can use just as many parameters as are necessary, without being forced to use a whole sub-image generated by the scaling function part of the wavelet transform.

Generally, 2D colour spaces will generate aliasing in the frequency domain because of artificial boundaries generated by the colour transform. Here, we use a novel stretched chromaticity space that has no such boundary. The use of this colour space removes a ringing effect generated by boundaries in other colour spaces.

To further shrink the number of parameters, we determine the Singular Value Decomposition (SVD) of the DCT coefficients. We found that we could reduce the number of coefficients in the SVD basis down to 8 without affecting performance.

What this series of steps generates is a universal SVD basis: universal in the sense that for any image or video frame, the illumination has essentially been normalised into the same, white illumination. Then we can project any new frame into the same space and intercompare correctly. Generation of the basis set is carried out offline. Generation of the coefficients for any new video is carried out online and since the basis set is so small, hierarchical clustering of videos is very fast. Output consists of video clusters plus their description in terms of a very small feature vector that can readily be used for subsequent indexing.

The set of steps described above was arrived at by a process of incremental determination of what worked best. The video frame feature described does very well at capturing colour information in a frame. Results for clustering are compared to a well-known technique and are found to greatly outperform the older method: keyframes generated form a far more succinct representation of videos and a visual story that agrees much more with a human-generated judgement of what the video contents consists of.

In $\S 2$ we present a description of a well-known method due to Ferman and Tekalp that is used to generate keyframes [11], since we intend to compare to this standard method as a means of judging success of our new method. Then we describe and justify the series of steps we use.

In $\S 3$ we set out the physics-based justification for the colour-channel normalization used here, and define the stretched chromaticity space.

Section 4 gives our clustering strategy. We use a 2-stage algorithm that in the first instance keeps temporal order to generate an unsupervised number of clusters, and then in a second pass attempts to group similar shots.

Section 5 states results on publicly available videos and shows the remarkable improvement in compactness of video summarization generated here. Section 6 states conclusions.

\section{Description of Previous Methods and Motivation for New Method}

Ferman and Tekalp [11] applied a clustering approach to intra-shot keyframe selection, based on the average colour histogram [25] and an intersection histogram [10]. The average histogram is indeed simply the average of all histograms in the shot. The intersection histogram is a generalization of the intersection of two image histograms - intersection is a rephrasing of histogram difference in an $\mathrm{L}_{1}$ norm [19]. Other algorithms advanced have used comparison to the first frame in a shot using colour and motion [23], accumulating frame differences [15], algorithms based on geometric metaphors, e.g. [3], and other approaches but here we mean to set out an algorithm that captures the salient colour features in a frame 
more effectively.

In [11], Ferman and Tekalp extract keyframes by clustering, and then go on to prune as well as augment the number of frames identified as keyframes, using a number of criteria. Firstly, since lighting changes can bias the average histogram adversely, outliers with respect to luminance are added to the list of keyframes. Secondly, clusters that contain only a few frames are eliminated. Thirdly, clusters with centers closer in colour distance than a threshold $T_{C}$ are merged. Finally, within each cluster, if the cluster radius exceeds $T_{C}$ two representative frames are chosen that are (1) closest to the center, and (2) farthest from the center. The authors use the Y-Cb-Cr colour space.

We have found that applying some of the techniques above yields poor results for keyframe extraction in quite common situations. For example, we considered the sequence illustrated by a few frames in Fig. 1 and found that lighting changes seen there as the child steps out of the shadows confound the algorithm in [11]. The method in [11] applied to such real outdoor lighting changes from shadowing can produce many keyframes, when in fact we wish to see only one.

In this paper we set out a method that performs correctly under this common lighting difficulty and moreover performs much better on all videos we have tried. Here, we utilise knowledge about colour formation for lighting and surfaces, from physics-based vision, to cast the clustering problem into the form of projection of frames onto a basis derived offline. This basis is precomputed from a large training set and then applies equally to all subsequent videos.

In this Section, we set out the series of steps used to create a very effective feature, and in the next Sections amplify on the introduction here in detail. The overall algorithm in displayed in Fig. 2.

Lighting is first discounted by normalization of colour-channel bands [8]. This step approximately but effectively removes dependence on both luminance and lighting colour. Then image frames are moved into a modified chromaticity colour space. As well as reducing the dimensionality of colour to 2, since chromaticity involves a ratio of colour bands this also has the effect of removing shading and intensity. In order to make the method fairly robust to camera and object motion, and displacements, rotations, and scaling, we go over to a 2D histogram derived from DC components of frames. In this paper we use $128 \times 128$ histograms. Chromaticity histograms are then compressed - i.e., we treat the histograms as images (see [20,8]). Here, we use a wavelet-based compression because this tends to strike a balance between simple low-pass filtering and retaining important image details [8]. Using a 3 -level wavelet compression we arrive at $16 \times 16$ histograms.

However, we found that compression of histograms could be improved if the histograms are first binarised, i.e., entries are replaced with 1 or 0 . The rationale for this step is that chromaticity histograms are a kind of colour signature for an image, similar to a palette. In work involving recovering the plausible illuminant from pixel values in an image [12] it was found beneficial to utilise this kind of chromaticity signature. We do not apply a threshold to binarise, and no colour in the image is considered less important than any other; we replace any nonzero bin by a 1 . In extensive tests, we found that inserting steps for countering the effects of noise, such as applying Gaussian smoothing to the 2D histograms, or using a threshold, had essentially no effect on results so these steps were then not included. Here, the step of binarising the histogram not only reduces the computational burden, since true chromaticity histograms need not be computed, but also has the effect of producing far fewer negatives in the compressed histogram. As a signature, then, we take the 2D compressed image of a binarised histogram for chromaticity.

Finally, we found that one further step could substantially improve the energy compaction of the representation: we carry out a $16 \times 16$ Discrete Cosine Transform (DCT) on the compressed $16 \times 16$ histogram. After zigzag ordering, as shown in Fig. 3 we keep 21 DCT coefficients: this corresponds to the first 6 anti-diagonals from the top-left corner of the DCT image. By using a DCT low-pass filtering, one can tailor the number of parameters finely by choosing just sufficient for a good representation of the colour content of a frame. 
The main utility of colour-channel normalization and then chromaticity space is that every image approximately goes over into the same lighting conditions. That is, we perform a kind of universal normalization of images. In fact, it was shown that going further in this direction and iterating the steps (1) colour-channel normalization, and (2) forming the chromaticity, which is itself a kind of colour normalization, produced excellent results for image indexing [13]. There, it was determined that about five iterations produced convergence, using such an iterated "comprehensive" normalization. Thus, here, we are going about one-fifth of the way towards the optimum, but still with good results and far less computation.

Since every image lives in approximately the same lighting, after our normalization steps, we can in fact precompute a basis for the DCT 21-vectors, offline, that can then be reused for any new image or video. Here we find a basis set by the SVD of the DCT 21-vectors (cf. [1], in which a basis set for DCT-transformed log colour-ratios was found using an SVD and [18] which used SVD on motion vectors and was considerably more complex than the method presented here). We found that 8 components in the new basis represent the entire DCT vector very well: 8 eigenvectors account for $99.6 \%$ of the variance. In [5] we found that energy compaction worked better using a spherical chromaticity of the type $(r, g)=(R, G) /\left(R^{p}+G^{p}+B^{p}\right)^{1 / p}$, rather than the usual linear one $(r, g)=(R, G) /(R+G+B)$. Here, we improve matters further by going over to a $2 \mathrm{D}$ stretched chromaticity space which has the advantage of utilising all bins in the histogram and also removing histogram edges.

Thus the method we set out here is to precompute a set of 8 basis vectors, once and for all, and then form the 8 -vector coefficients for any video frame with respect to this basis. Then keyframe extraction by clustering can be carried out very efficiently, using only 8 -component vectors, and need not be performed only intra-shot, as in [11]. In fact, we can present an entire video sequence to a clustering method, or just a small sequence, and still find keyframes.

Abrupt cuts certainly form boundaries between shots, but gradual transitions can also be simply identified as regions with a fast derivative of keyframe number - if many keyframes are produced over a short time period then, while this could be due to very fast scene changes, the most likely explanation is that a gradual transition or cut has taken place. Thus, rather than keeping these transitions, we discard them.

Each keyframe is associated with a length- 8 feature vector of basis coefficients. Thus video search, retrieval, and browsing are a fast and simple matter once keyframe production has occurred.

The clustering method we adopt is a variant of hierarchical clustering [9], adapted to video. Firstly, only adjacent frames are merged, as we wish to maintain the temporal order of keyframes in a video summary (as opposed to discarding temporal order, as in [14]). This permits an efficient data structure for the clustering tree and distance matrix. Also, an initial clustering is followed by an additional step on clusters found, marking some keyframes as representing nearrepeated shots.

This efficiently provides a further pruning step that has the effect of merging similar shots. The result is a set of frames that provide both an intra- and inter-shot representation of the video. Further, the second, cluster-merging step, automatically provides the structure for a graph-based representation of the video.

The threshold we use to control the number of clusters is the variance ratio; this is efficient to compute and is adaptive to different videos. We can efficiently calculate variance for all sub-clusters (maintained in the hierarchical tree structure). In [6] we used a threshold based on the ratio of intra-cluster variance to total variance. Here we find that a threshold based on the ratio of intra-cluster variance to variance of the parent node performs best. We determined that a universal threshold of $T_{V R}=0.25$ variance ratio performs well.

While boundary detection is simply quantified, testing performance for keyframe extraction involves a comparison of keyframes generated to those that should be generated, and for ground truth we must here rely on human opinion. With 
that proviso, we find that the method presented here does agree well with human notions of what frames most expressively summarise a video sequence in that the extracted keyframes agree much better with those generated manually by unbiased observers.

\section{Illumination Change and Normalization}

\subsection{Pixel values under illumination change}

The motivation for colour-channel normalization lies in making images approximately independent of illumination; such a normalization has the concomitant effect of transforming every image into the same illumination domain.

To understand how colour normalization effects illuminant independence, firstly we note that several justifications can be made [4] for a diagonal model for illumination change: colour values for pixels approximately undergo independent multiplicative changes in each colour channel R, G, and B, that are independent of retinal coordinates.

For consider pixel values $\boldsymbol{\rho}^{x}$ at camera retinal position $\boldsymbol{x}$. Under lighting $E(\lambda)$, if the light impinges on a Lambertian surface with reflectance function $S(\lambda)$ from direction $\boldsymbol{a}$, the RGB camera value produced is

$$
\boldsymbol{\rho}^{x}=\boldsymbol{a} \cdot \boldsymbol{n}^{x} \int E(\lambda) S^{x}(\lambda) \boldsymbol{q}(\lambda) d \lambda
$$

where $\boldsymbol{q}(\lambda)$ are three camera sensitivity functions, and $\boldsymbol{n}^{x}$ is the surface normal at position $x$. Now, according to Borges [2], the usual factor model approximation used in computer graphics can be employed:

$$
\begin{gathered}
\rho_{k}^{x} \simeq \boldsymbol{a} \cdot \boldsymbol{n}^{x} s_{k}^{x} e_{k} / \sigma_{k}, k=1 . .3, \quad \text { with } \\
s_{k}^{x}=\int S^{x}(\lambda) q_{k}(\lambda) d \lambda, e_{k}=\int E(\lambda) q_{k}(\lambda) d \lambda, \sigma_{k}=\int q_{k}(\lambda) d \lambda
\end{gathered}
$$

providing the light is fairly white. Therefore under a lighting change $E \rightarrow E^{\prime}, \boldsymbol{a} \rightarrow \boldsymbol{a}^{\prime}$, pixel values transform according to

$$
\rho_{k}^{x} \rightarrow \rho_{k}^{x \prime}=\left(e_{k}^{\prime} / e_{k}\right) \rho_{k}^{x}
$$

Hence normalising each colour channel, in $\mathrm{L}_{1}$ or $\mathrm{L}_{2}$ norm, effectively removes the lighting. This is so because the ratio $\left(e_{k}{ }^{\prime} / e_{k}\right)$, which effectively captures the lighting change, is removed if the entire collection of pixel values $x=1 . . N^{2}$ is divided by the sum (or mean), say, separately for each channel $k$, with resulting greyscale image in channel $k$ the same for light $E^{\prime}$ as for light $E$.

Suppose we do divide every pixel's $k$ th channel value $\rho_{k}^{x}$ by the mean $\mu_{k}$ of all such values:

$$
\mu_{k}=(1 / N) \sum_{x}\left(\boldsymbol{a} \cdot \boldsymbol{n}^{x}\right) s_{k}^{x} e_{k} / \sigma_{k} .
$$

Then pixels in the $k$ th channel are normalised to

$$
\begin{aligned}
\rho_{k}^{x} \rightarrow \rho_{k}^{x} / \mu_{k} & =\frac{\boldsymbol{a} \cdot \boldsymbol{n}^{x} s_{k}^{x} e_{k} / \sigma_{k}}{\left(e_{k} / \sigma_{k}\right)(1 / N) \sum_{x}\left(\boldsymbol{a} \cdot \boldsymbol{n}^{x}\right) s_{k}^{x} e_{k}} \\
& =\frac{\boldsymbol{a} \cdot \boldsymbol{n}^{x} s_{k}^{x}}{(1 / N) \sum_{x}\left(\boldsymbol{a} \cdot \boldsymbol{n}^{x}\right) s_{k}^{x}}
\end{aligned}
$$


That is, in order to cast (5) into the same form as eq. (2), we must use an effective lighting term

$$
\widetilde{e_{k}}=N \sigma_{k} / \sum_{x} \boldsymbol{a} \cdot \boldsymbol{n}^{x} s_{k}^{x}
$$

Now consider the common "gray-world" assumption, whereby the average colour of surfaces in an image is assumed to be given by

$$
(1 / N) \sum_{x} \boldsymbol{a} \cdot \boldsymbol{n}^{x} s_{k}^{x}=\sigma_{k}
$$

i.e., the world is "gray" on average. Hence what we are doing in dividing by the mean is transforming every image into the same, common lighting $\widetilde{e_{k}}=1$, under a gray-world assumption — every image goes into the same lighting.

Going over to a 2D chromaticity $(r, g)=(R, G) /(R+G+B)$ removes the shading term $\boldsymbol{a} \cdot \boldsymbol{n}^{x}$ as well, and thus removes the effect of lighting direction. In [6], as discussed in $\S 4$, we actually used a spherical chromaticity $(r, g)=$ $(R, G) / \sqrt{R^{2}+G^{2}+B^{2}}$. The reason for going over to this space from the usual one is that linear chromaticity presents an edge in the resulting histogram, and this edge creates a ringing effect in a Fourier domain version. The spherical chromaticity mitigates this problem.

Here, we improve matters further by replacing the spherical chromaticity by a stretched chromaticity space similar to that in $[17]^{1}$,

$$
\begin{aligned}
& (r, g)=(R, G) /(R+G+B) \quad,
\end{aligned}
$$

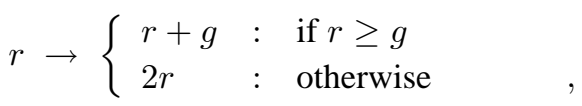

$$
\begin{aligned}
& g \rightarrow\left\{\begin{array}{lll}
2 g & : & \text { if } r \geq g \\
r+g & : & \text { otherwise }
\end{array}\right.
\end{aligned}
$$

which also eliminates the edge effect and has the advantage of making use of all bins in the histogram.

\subsection{Universal basis}

Because we reduce all images to approximately the same colour domain, we can calculate offline a basis on which to project each video frame. In [8] we used a wavelet-based compression method, applied to histograms as if they were images. The use of a wavelet-based compression reflects a trade-off between complexity and expressiveness. We found that the compression strategy used allowed important features in a histogram to persist in the smaller version, whereas simply using a coarse binning did not. A further low-pass filter was applied by forming the DCT image of the resulting histogram, zig-zag coding, and truncating. However, that method can be improved in several substantive ways. Firstly, consider the $128 \times 128$ 2D chromaticity histogram in Fig.4(b) that arises from the video frame in Fig.4(a). The original image essentially consists of only a few uniformly-coloured patches and so produces a histogram comprising a few sharp spikes. As a consequence, the wavelet-based compression gives rise to some negatives, as in Fig.4(c). This is a typical result - the Figure is an example of behaviour that can often crop up in a great many test images we have considered.

A more well-behaved compression results if, instead, we replace the histogram with a binarised one, i.e., with entries either 0 or 1 . In a sense, this shows a kind of colour signature for the image, like a colour palette. Now the wavelet-

\footnotetext{
${ }^{1}$ This chromaticity space is similar to that in [17], but properly produces the range $\{[0 . .1],[0 . .1]\}$, rather than $\{[0 . .1],[0 . .2]\}$.
} 
compressed histogram appears as in Fig.4(d): the percentage of negative counts in the histogram has gone from $8 \%$ in Fig.4(c) to only 1\% in Fig.4(d). Again, this is a typical result. Here we use a Daubechie 4-tap wavelet.

As well, the DCT produces better energy compaction if we go over to a nonlinear definition of chromaticity. The reason is that, since linear chromaticity obeys $r+g \leq 1$, there can exist a straight diagonal edge in a chromaticity histogram, as in Fig.5(b), which arises from the image Fig.5(a). The DCT image for this histogram shows a ringing effect, as in Fig.5(c). This is a typical result — the effect arises as the rule, not the exception, in images we have studied. Previously, we made use of a spherical definition of chromaticity with the straight edge replaced by a circle, with the effect of mitigating the ringing [5]. Here, we go one better and remove the histogram edge entirely, via the transform (8) to a stretched chromaticity space; in all images we have studied this 2D space has provided the best energy compaction.

Figs.5(e,f) show the stretched chromaticity colour signature and the resulting DCT image for the compressed histogram: the DCT is quite concentrated.

As a final step, we form the SVD basis for the first 21 DCT coefficients, in zigzag order, for a large training set of images. We found that 8 basis vectors suffice quite well for DCT images of videos we examined. Projected onto this subspace, the $21 \mathrm{DCT}$ values for each frame result in an 8 -component feature vector.

\section{Clustering Intra- and Inter-Shots}

\subsection{Clustering strategy}

Fig. 2 shows the clustering scheme employed, using the 8 -vector features outlined above. Although these vectors are longer than those used in [11], and hence require more computation for a distance calculation, they carry much more information about the video than do the 2-vectors used there since they are grounded in a physics-based vision approach that arises from the underlying principles of colour formation.

To begin with, we compute the 8 -vector features for each frame, projecting onto the pre-computed basis. This step represents the majority of time used by the algorithm for each new video - however, it can be carried out offline for non-realtime applications, and we do project onto a pre-determined basis set. Because the feature space is relatively lowdimensional, and especially because we merge only adjacent frames, frame clustering is very fast. With buffering, this means that we could effectively carry out clustering on data from a temporal window of streaming video, presenting only keyframes to the user.

Only adjacent clusters are merged. The threshold used is the Variance Ratio = (intra-cluster variance)/(variance of parent node). As well, we apply a minimum variance threshold. The reason for this is that if the entire video consists of only one scene, the ratio becomes meaningless. A minimum variance threshold identifies such uniform videos. Fortunately, these kinds of videos contain total variance that is usually much smaller than those of complex videos (by about a factor of 20); thus we are able to select a global threshold that can work on most types of videos - here we used $T_{\min }=0.01$.

We also considered what effect basing the initial clustering step only on adjacent frames had on the optimality of the clustering. Generally, it is straightforward to correct erroneous decisions in hierarchical clustering by following an initial placement into $k$ clusters by an iterative relocation step to refine the results for the $k$ clusters (BIRCH is one such algorithm [24]. ) We found that a further iterative step for optimization usually re-arranged only a small number of frames. Thus using adjacent frames in a hierarchical clustering already produces an adequate partitioning.

Finally, we carry out a second clustering step, on the clusters found. This has the effect of dropping whole shots that are near-repeats, as in the sequence ABCADEF. 


\subsection{Clustering algorithm}

In detail, the steps used are as follows:

1. Perform bottom-up hierarchical clustering using minimum $\mathrm{L}_{2}$ distance between cluster means. Only adjacent clusters are merged. Using distances between cluster averages generates fairly spherical clusters; each cluster is formed from contiguous frames. This operation is fast.

Other cluster-generation strategies would produce different trees. For example, another approach would be to merge clusters based on the minimum distance between a point in the first cluster and a point in the second cluster. This is likely comparable to simple frame-difference algorithms since two consecutive frames are probably closest to each other. Most transitions in our test videos are gradual transitions, for which the next frame is added to the previous cluster (we use a forward-moving algorithm). This strategy typically creates long, thin clusters and we would obtain an entirely lop-sided tree.

2. Top-down, extract clusters that satisfy both the variance ratio threshold (intra-cluster variance) $<$ (parent node variance) $* T_{V R}$ and the minimum variance threshold. This gives us an initial set of clusters.

Here we use variance $V$ as a measure of similarity, and consider only the total variance $V$ of a set of $D$-dimensional vectors, with $D=8$ (and not a more complex measure, such as a covariance matrix): $V=\sum_{i=1}^{D} \mathrm{E}<\left(v_{i}-\mathrm{E}<\right.$ $\left.v_{i}>\right)^{2}>$, where $\mathrm{E}<>$ is expectation value. All top-level clusters whose variance is less than $T_{V R}$ times that of the parent node are considered self-similar enough to represent one shot. This relative measure provides the ability to adapt to different videos. For instance, videos that have a wide range of chromaticity signatures will tend to have scenes that also contain a wide range of chromaticity signatures, and vice versa for videos with a small range of chromaticity signatures. However, intra-shot variances are usually significantly lower than inter-shot variances, and Variance Ratio captures this relative character. It also allows automatic determination of the number of shots.

3. Now we have an initial set of clusters. However, there are still two shortcomings: dissolve transitions and errors from the greedy hierarchical clustering. A dissolve transition may create many clusters because the chromaticity signature changes sharply within the transition. Since the clusters are spherical, the transition produces many clusters within a short time frame.

Another artifact is that sometimes two similar adjacent clusters may be produced. This happens when a scene got split up in the early rounds by the greedy hierarchical clustering algorithm.

Therefore we carry out another round of merging adjacent clusters that satisfy the thresholds.

4. In order to identify gradual transitions, and discard them, all contiguous clusters of size $\leq 3$ are merged and marked as a transition. This works well for dissolve transitions but not for wipes (the latter are discussed below, in $\S 6$ ).

5. Clustering has now produced clusters that maintain temporal order. However, we may not wish to be faithful to temporal order so that similar, near-repeat shots are not repeatedly included in the summarization. Therefore we hierarchically merge non-adjacent, non-transition clusters that result in lower variance: if cluster AB's variance is lower than that of either cluster A or B, then the two are merged. Intuitively, this means that we only perform a merge if it improves the self-similarity of the cluster. 
6. Finally, each non-transition cluster is represented by the single keyframe closest to the cluster's centroid. Note that information for all clusters (frame ranges, variance, variance ratio, transition) is still available for any further development.

\section{Results}

We implemented the average histogram plus intersection histogram algorithm set out in [11], with parameters set as specified there. Table 1 shows results for the present basis projection algorithm compared to results using [11]. Here both methods are compared to "ground truth", which was a set of keyframes for each video extracted manually by an unbiased observer.

Not only are the summarizations much more succinct for the algorithm set out here, they are correctly unresponsive to changes in illumination, and also agree very well with human summarizations. In general, even given perfect transition detection, the method [11] generates too many keyframes whereas the present method does not; without perfect transition detection, [11] misses many more keyframes than the present method.

Visually, the success of the new method is very convincing: since the new method is meant to discount lighting changes, we first consider its application to the video for Fig. 1 (http://www.cs.sfu.ca/ mark/Ivc03/child.mpg). As shown in Fig. 6, we expect a correct output to consist of only a single frame; the present algorithm indeed generates only one frame, but the previous method generates 4. Results for a more typical video are shown in Fig. 7: here a sports video (http://busboy.sped.ukans.edu/campus/movies/football/football.mpg) contains a good deal of quick action. The human observer has extracted 5 keyframes for this video. The present algorithm generates 5 keyframes as well, but of these one of the keyframes in the ground truth set is missed, and one is replaced by (an approximately similar) pair of keyframes. In comparison, the method in [11] generates 18 keyframes, far too many. Of these, none are misses but 13 are redundant in that such keyframes (or close ones, which are also allowed) do not occur in the ground truth set.

For a video which contains sudden lighting changes and a changing background (Basketball, in Fig. 8) the discrepancy is even more apparent: whereas we generate 8 keyframes (as opposed to the correct number which is 10) with 1 missed and 1 redundant keyframe, the older method generates 132 keyframes, of which 122 are redundant.

Other summarization results as per Table 1 are similar. These results are further summarised in Table 2, where in this context Recall and Precision have the meanings

$$
\begin{aligned}
\text { Recall } & =\frac{\text { \#Correct Generated }}{\text { \#Actual Keyframes }} \\
\text { Precision } & =\frac{\text { \#Correct Generated }}{\text { \#Keyframes Generated }}
\end{aligned}
$$

where "Actual" is the set of ground truth keyframes as judged by an unbiased human observer. Clearly, the present algorithm has superior performance to the previous method, in some cases overwhelmingly superior. Overall, whereas the previous method has very high Precision, its Recall is very low; and the method presented here has considerably greater Recall while maintaining a high Precision.

However, we did find that wipe transitions could become clusters on their own. In this situation the extracted keyframe is a "half-wipe" frame. Alternatively, a wipe transition may get shared between two clusters - the first half of the wipe transition is merged into the shot before and the second half is merged into the shot after. This occurs because a wipe 
transition contains chromaticity signatures of both shots and the spherical nature of the clusters naturally divides the transition into two. We intend to pursue this problem, applying an auxiliary method to detect wipes (see, e.g., that in [7]).

Another issue is that videos that contain fast camera motion may not be properly segmented since such motion may be interpreted as a gradual transition.

Misses mostly occur when the new scene's colours do not vary enough from the previous scene. An example would be when the previous shot persists in a smaller version, inside the current shot. Colours that are very simple, as in computer graphics generated scenes or animations, have such problems but this is likely unavoidable for any method used.

Original videos, plus results, may be viewed at the web site: http://www.cs.sfu.ca/ mark/ftp/Ivc03/ .

\section{Conclusions}

In this paper we have set out a new low-dimensional video frame feature that is approximately resistant to changes in lighting, and also is based on a chromaticity space that effectively removes shading. In the space of binarised 2D histograms of chromaticity, we first compress histograms as if they were images, using a wavelet-based compression. Zig-zag coding and discarding all but the first few anti-diagonals reduces the dimensionality of feature vectors down to 21 , and a further SVD steps produces a universal basis in a space with dimension only 8 . The low dimensionality allows us to carry out an efficient clustering scheme, for temporally-ordered clusters. We base a hierarchical clustering method on the ratio of intra-cluster variance to that of the parent node, merging only adjacent clusters, and then follow with a second round of clustering to fuse near-repeat shots.

Using the present method, video summarization results produced are excellent, yielding a very succinct set of keyframes that agrees well with human expectations with almost no redundancy and very few misses. The success of the method depends on both the new approach to colour histograms and the multi-stage hierarchical clustering algorithm. Since the clustering method uses fixed, non-adaptive thresholds, there are no parameters to tune. The reduction of every video to the same lighting is the mechanism that allows the same thresholds to be used for any input.

\section{Acknowledgements}

The authors are indebted for support to the Hewlett-Packard Corp. and to Lightseer Ltd. (M.S.D), and to the Natural Sciences and Engineering Research Council of Canada for a Postgraduate Scholarship (J.A.) and for Operating Grant 31-611280 (M.S.D.).

\section{References}

[1] J. Berens and G.D. Finlayson. Log-opponent chromaticity coding of colour space. In ICPR '00, 2000. To appear.

[2] C.F. Borges. Trichromatic approximation method for surface illumination. J. Opt. Soc. Am. A, 8:1319-1323, 1991.

[3] D. DeMenthon, V. Kobla, and D. Doermann. Video summarization by curve simplification. In ACM MM98, 1998.

[4] M. S. Drew, J. Wei, and Z.N. Li. Illumination-invariant image retrieval and video segmentation. Pattern Recognition, 32:1369-1388, 1999. 
[5] M.S. Drew and J. Au. Video keyframe production by efficient clustering of compressed chromaticity signatures. In ACM Multimedia '00, pages 365-368, 2000. http://www.cs.sfu.ca/ mark/ftp/AcmMM00.

[6] M.S. Drew and J. Au. Video summarization by efficient clustering of compressed chromaticity signatures. In The First IEEE Pacific-Rim Conf. on Multimedia, 2000. http://www.cs.sfu.ca/ mark/ftp/Ismip00.

[7] M.S. Drew, Z.-N. Li, and X. Zhong. Video dissolve and wipe detection via spatio-temporal images of chromatic histogram differences. In International Conference on Image Processing: ICIP '00, pages III 929-932, 2000. http://www.cs.sfu.ca/ mark/ftp/Icip00/icip00.pdf.

[8] M.S. Drew, J. Wei, and Z.N. Li. Illumination-invariant color object recognition via compressed chromaticity histograms of color-channel-normalized images. In ICCV98, pages 533-540. IEEE, 1998.

[9] B.S. Everitt. Cluster analysis. Halsted Press, 3rd edition, 1993.

[10] A.M. Ferman and A.M. Tekalp. Multiscale content extraction and representation for video indexing. In SPIE Multimedia Storage and Archiving Systems II, 1997.

[11] A.M. Ferman and A.M. Tekalp. Efficient filtering and clustering methods for temporal video segmentation and visual summarization. J. Vis. Commun. \& Image Rep., 9:336-351, 1998.

[12] G.D. Finlayson, P.M. Hubel, and S. Hordley. Colour by correlation. In Fifth Color Imaging Conf., pages 6-11, 1997.

[13] G.D. Finlayson, B. Schiele, and J.L. Crowley. Comprehensive colour image normalization. In ECCV98, 1998.

[14] A. Girgensohn and J. Boreczky. Time-constrained keyframe selection technique. In IEEE MM Sys., pages 756-761, 1999.

[15] A. Hanjalic, M. Ceccarelli, R.L. Lagendijk, and J. Biemond. Automation of systems enabling search on stored video data. In SPIE Storage and Retrieval for Image and Video Databases V, pages 427-438, 1997.

[16] J. R. Kender and B. L. Yeo. Video scene segmentation via continuous video coherence. In CVPR '98, pages 367-373, 1998.

[17] D. Paulus, K. Horecki, and K. Wojciechowski. Localization of colored objects. In International Conference on Image Processing: ICIP '00, pages Vol III 492-495, 2000.

[18] E. Sahouria and A. Zakhor. Content analysis of video using principal components. IEEE Trans. Circ. Sys. Vid. Tech., 9:1290-1298, 1999.

[19] M.J. Swain and D.H. Ballard. Color indexing. Int. J. Comput. Vision, 7(1):11-32, 1991.

[20] J. Wei, M.S. Drew, and Z.N. Li. Illumination invariant video segmentation by hierarchical robust thresholding. In Electronic Imaging '98: Storage and Retrieval for Image and Video Databases VI, pages 188-201. SPIE Vol. 3312, 1998.

[21] B.-L. Yeo and M.M. Yeung. Classification, simplification and dynamic visualization of scene transition graphs for video browsing. In SPIE Storage and Retrieval for Image and Video Databases VI, 1998. 
[22] M.M. Yeung and B. Liu. Efficient matching and clustering of video shots. In ICIP '95, pages 338-341, 1995.

[23] H.J. Zhang, S.Y. Tan, S.W. Smoliar, and Y. Gong. Video parsing, retrieval and browsing: An integrated and contentbased solution. In ACM Multimedia '95, pages 15-24, 1995.

[24] T. Zhang, R. Ramakrishnan, and M. Livny. BIRCH: an efficient data clustering method for very large databases. In ACM-SIGMOD Int. Conf. on Management of Data, pages 103-114, 1996.

[25] D. Zhong, H. Zhang, and S.-F. Chang. Clustering methods for video browsing and annotation. In SPIE Storage and Retrieval for Image and Video Databases IV, pages 239-246, 1996. 


\begin{tabular}{|c|c|c|c|c|c|c|c|}
\hline & \multicolumn{3}{|c|}{ Signatures } & \multicolumn{3}{|c|}{ HistInt } \\
\hline & & \multirow{2}{*}{$\begin{array}{c}\text { \#Keyframes } \\
\text { Generated }\end{array}$} & \multirow[t]{2}{*}{ Redundant } & \multirow[t]{2}{*}{ Missed } & \multirow{2}{*}{$\begin{array}{c}\text { \#Keyframes } \\
\text { Generated }\end{array}$} & \multirow[t]{2}{*}{ Redundant } & \multirow[t]{2}{*}{ Missed } \\
\hline Video (\#Frames) & Actual & & & & & & \\
\hline Football (560) & 5 & 5 & 1 & 1 & 18 & 13 & 0 \\
\hline Basketball (897) & 10 & 10 & 1 & 1 & 132 & 122 & 0 \\
\hline Child (30) & 1 & 1 & 0 & 0 & 4 & 3 & 0 \\
\hline Aqmov (801) & 6 & 4 & 0 & 2 & 36 & 30 & 0 \\
\hline Beachmov (463) & 4 & 3 & 0 & 1 & 24 & 20 & 0 \\
\hline Canmov (480) & 4 & 3 & 0 & 1 & 23 & 19 & 0 \\
\hline Capmov (487) & 5 & 5 & 0 & 0 & 12 & 8 & 1 \\
\hline Dbmov (441) & 8 & 8 & 1 & 1 & 24 & 17 & 1 \\
\hline Jazzmov (361) & 6 & 5 & 2 & 3 & 16 & 10 & 0 \\
\hline Prof (82) & 1 & 1 & 0 & 0 & 6 & 5 & 0 \\
\hline Steam (405) & 1 & 1 & 0 & 0 & 6 & 5 & 0 \\
\hline Walkmov (497) & 4 & 5 & 1 & 0 & 18 & 14 & 0 \\
\hline Aba (179) & 2 & 2 & 0 & 0 & 10 & 8 & 0 \\
\hline Nitobmov (580) & 4 & 7 & 3 & 0 & 18 & 14 & 0 \\
\hline Simpsons (2004) & 20 & 20 & 6 & 5 & 139 & 119 & 0 \\
\hline Total (8267) & 81 & 78 & 13 & 16 & 347 & 288 & 2 \\
\hline
\end{tabular}

Table 1:

\section{Table 1 Caption}

Keyframes generated, compared to algorithm in [11]. The correct number of keyframes over test videos totals 81 . "Signatures" indicates present algorithm. "HistInt" indicates method in [11], with $k=3$ and $T_{c}=3000$ as specified there. "Actual" is as judged by an unbiased human observer. 


\begin{tabular}{|c|c|c|c|c|c|c|c|}
\hline & \multicolumn{3}{|c|}{ Signatures } & \multicolumn{3}{|c|}{ HistInt } \\
\hline & & \multirow{2}{*}{$\begin{array}{l}\text { \#Correct } \\
\text { Generated }\end{array}$} & \multirow[t]{2}{*}{ Recall } & \multirow[t]{2}{*}{ Precision } & \multirow{2}{*}{$\begin{array}{l}\text { \#Correct } \\
\text { Generated }\end{array}$} & \multirow[t]{2}{*}{ Recall } & \multirow[t]{2}{*}{ Precision } \\
\hline Video (\#Frames) & Actual & & & & & & \\
\hline Football (560) & 5 & 4 & $80 \%$ & $80 \%$ & 5 & $100 \%$ & $28 \%$ \\
\hline Basketball (897) & 10 & 9 & $90 \%$ & $90 \%$ & 10 & $100 \%$ & $8 \%$ \\
\hline Child (30) & 1 & 1 & $100 \%$ & $100 \%$ & 1 & $100 \%$ & $25 \%$ \\
\hline Aqmov (801) & 6 & 4 & $67 \%$ & $100 \%$ & 6 & $100 \%$ & $17 \%$ \\
\hline Beachmov (463) & 4 & 3 & $75 \%$ & $100 \%$ & 4 & $100 \%$ & $17 \%$ \\
\hline Canmov (480) & 4 & 3 & $75 \%$ & $100 \%$ & 4 & $100 \% 9$ & $17 \%$ \\
\hline Capmov (487) & 5 & 5 & $100 \%$ & $100 \%$ & 4 & $80 \%$ & $33 \%$ \\
\hline Dbmov (441) & 8 & 7 & $88 \%$ & $88 \%$ & 7 & $88 \%$ & $29 \%$ \\
\hline Jazzmov (361) & 6 & 3 & $50 \%$ & $60 \%$ & 6 & $100 \%$ & $38 \%$ \\
\hline Prof (82) & 1 & 1 & $100 \%$ & $100 \%$ & 1 & $100 \%$ & $17 \%$ \\
\hline Steam (405) & 1 & 1 & $100 \%$ & $100 \%$ & 1 & $100 \%$ & $17 \%$ \\
\hline Walkmov (497) & 4 & 4 & $100 \%$ & $80 \%$ & 4 & $100 \%$ & $22 \%$ \\
\hline Aba (179) & 2 & 2 & $100 \%$ & $100 \%$ & 2 & $100 \%$ & $20 \%$ \\
\hline Nitobmov (580) & 4 & 4 & $100 \%$ & $57 \%$ & 4 & $100 \%$ & $22 \%$ \\
\hline Simpsons (2004) & 20 & 14 & $70 \%$ & $70 \%$ & 20 & $100 \%$ & $14 \%$ \\
\hline Total (8267) & 81 & 65 & $\overline{a v=86 \%}$ & av $=88 \%$ & $\overline{79}$ & $\overline{a v}=98 \%$ & $\overline{a v}=22 \%$ \\
\hline
\end{tabular}

Table 2:

\section{Table 2 Caption}

Results expressed as Recall and Precision: Recall is (\#Correct Generated)/(\#Actual Keyframes) and Precision is (\#Correct Generated)/(\#Keyframes Generated), with (\#Correct Generated) given by (\#Keyframes Generated) - \#Redundant). 


\section{Figure Captions}

Fig.1: Video with lighting change.

Fig.2: Basis generation and clustering algorithm.

Fig.3: Retention of low-pass coefficients after zigzag coding.

Fig.4: (a): Video frame. (b): Chromaticity histogram. (c): Compressed histogram. (d): Binarised histogram, compressed.

Fig.5: (a): Image. (b): Linear chromaticity. (c): Ringing effect occurs in DCT if chromaticity edge is sharp. (d): Stretched chromaticity. (e): Chromaticity signature: compressed binarised histogram of stretched chromaticity. (f): Absolute value of DCT shows energy compaction.

Fig.6: (a): Correct summarization of video child.mpg. (b): Signatures algorithm result. (c): Hist Int algorithm result.

Fig.7: (a): Correct: video football .mpg. (b): Signatures result. (c): Hist Int result.

Fig.8: (a): Correct: video basketball .mpg. (b): Signatures result. (c): Hist Int result. 


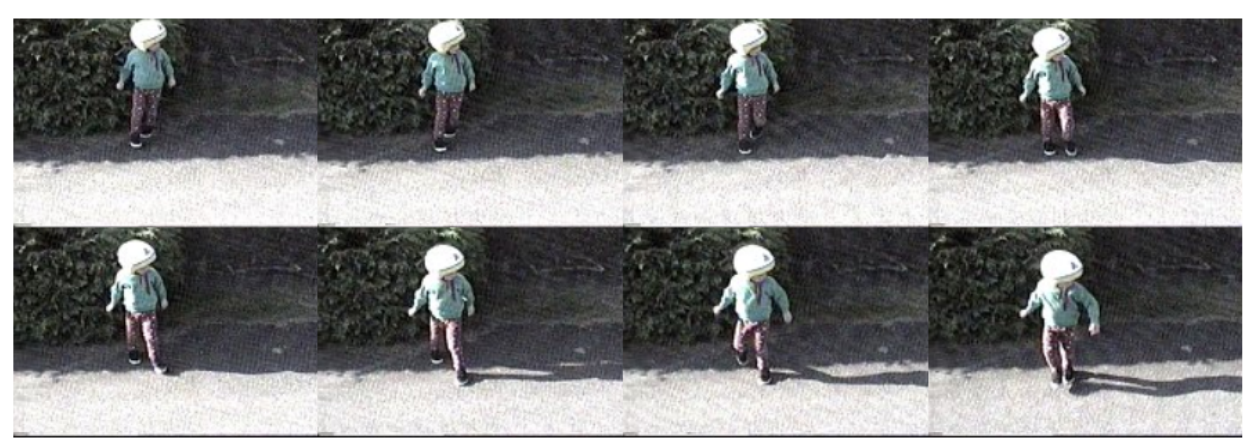

Figure 1: 


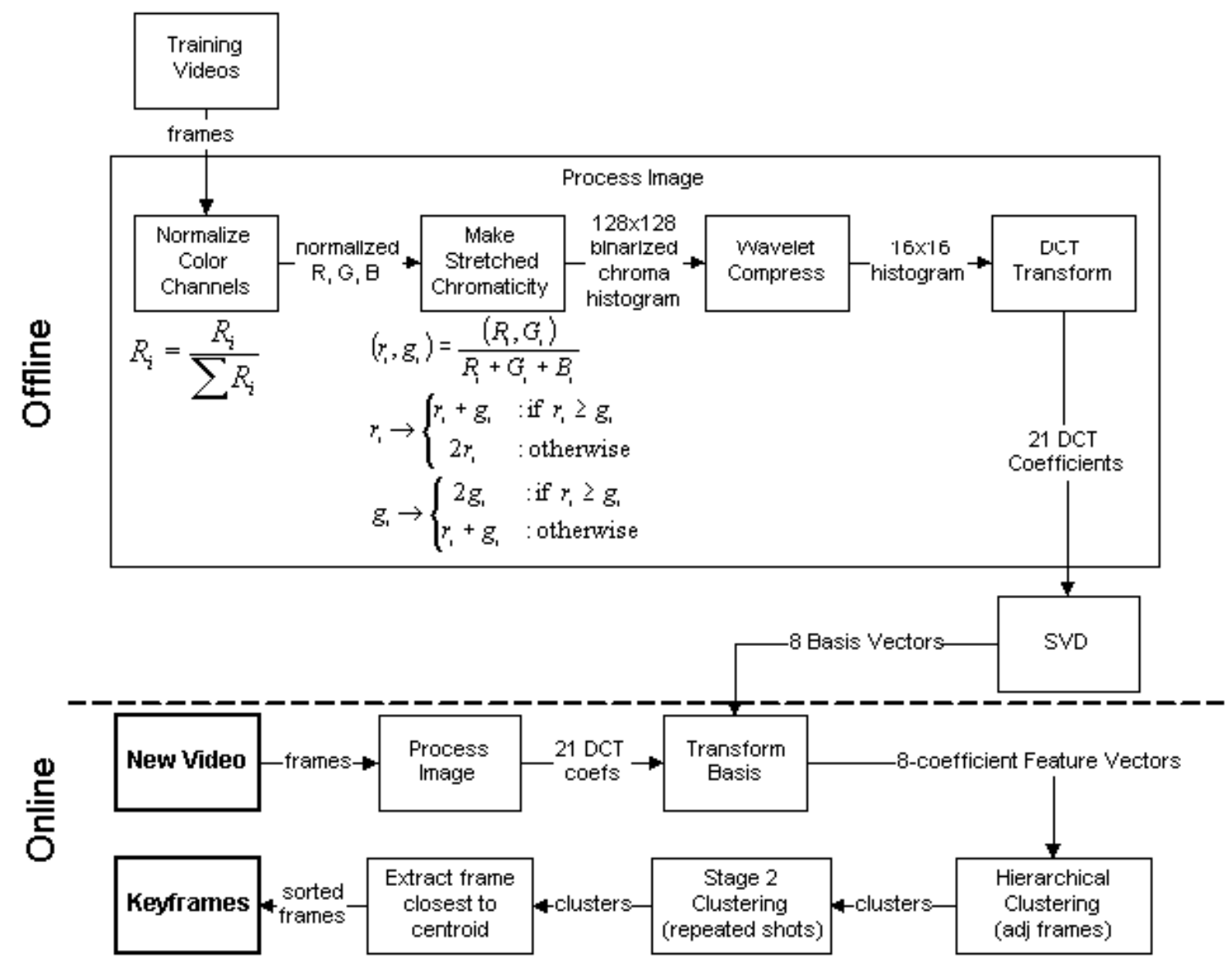

Figure 2: 


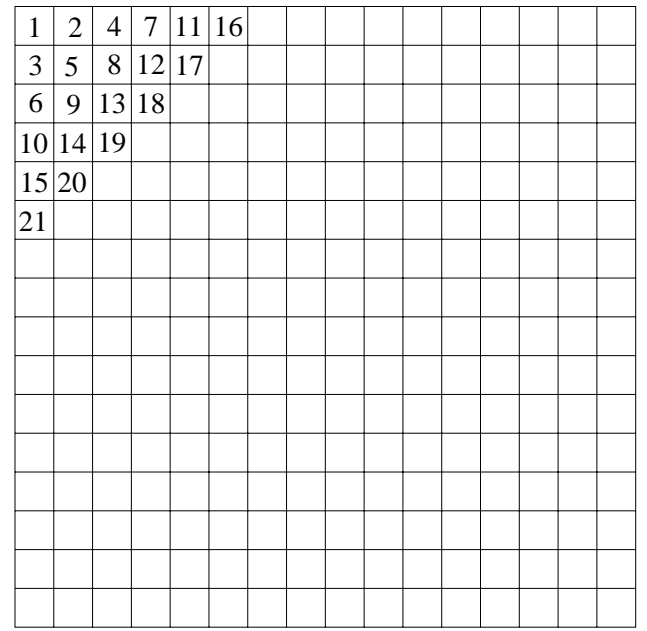

Figure 3:

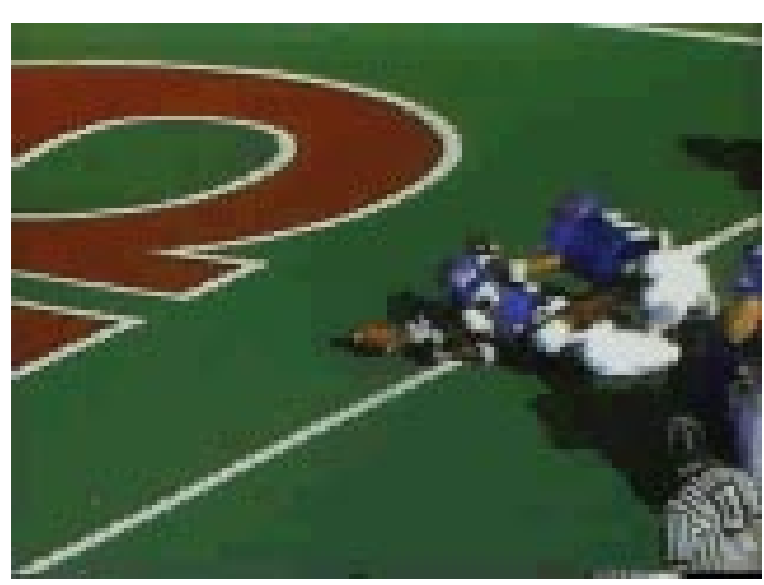

(a)

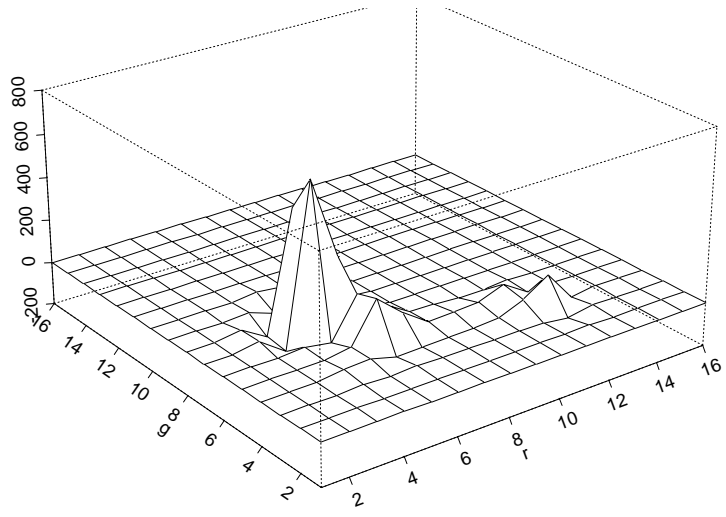

(c)

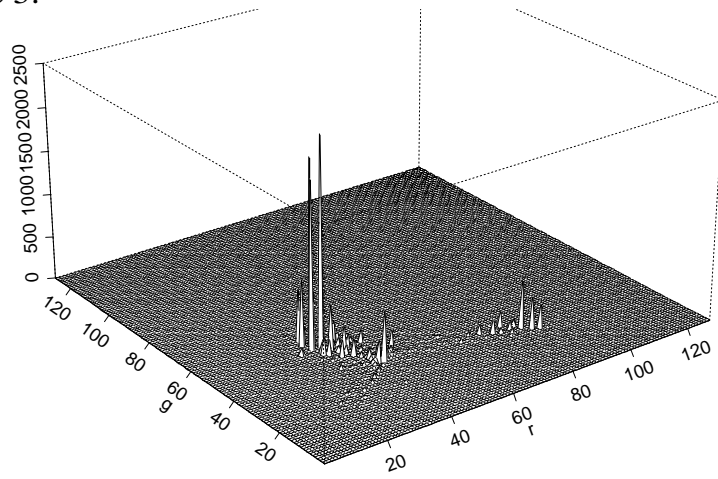

(b)

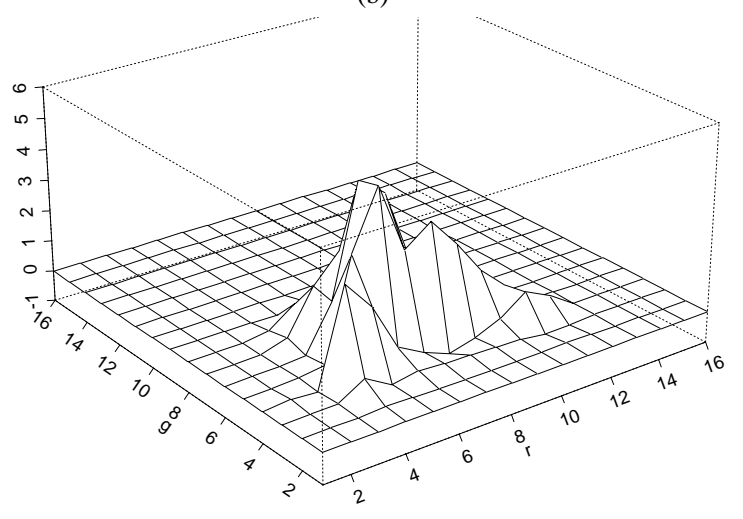

(d)

Figure 4: 


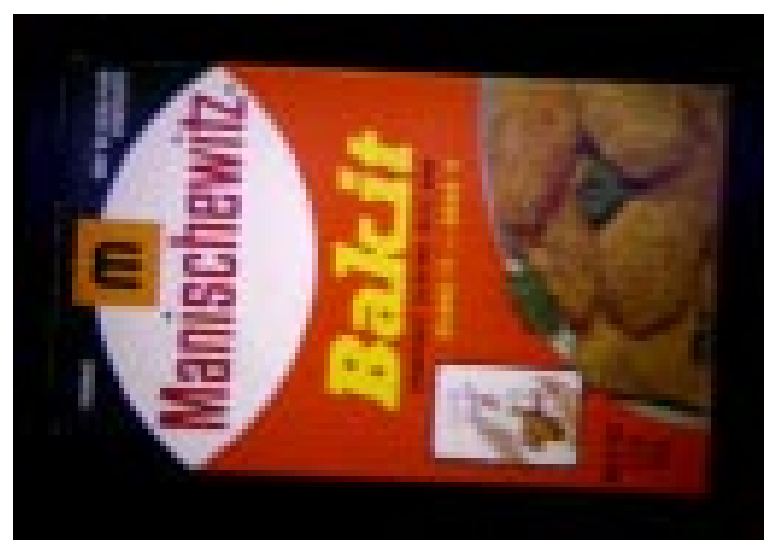

(a)

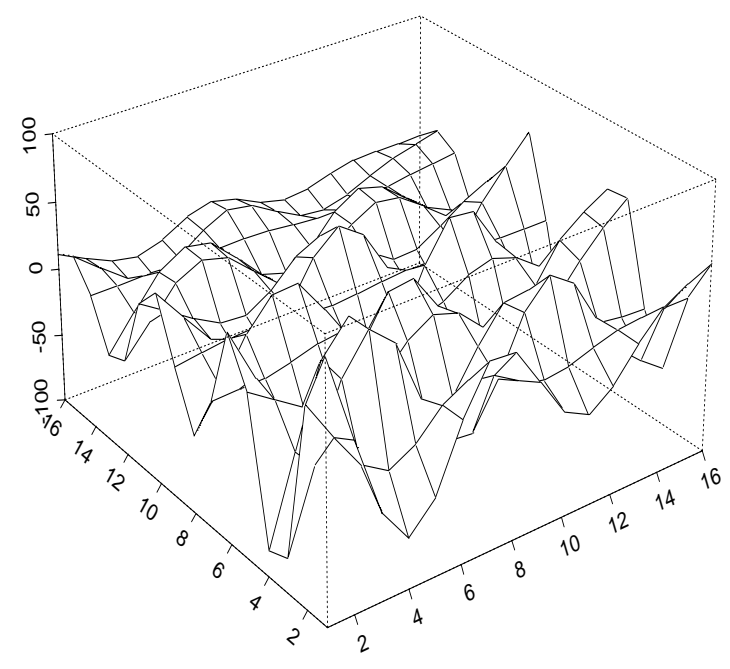

(c)

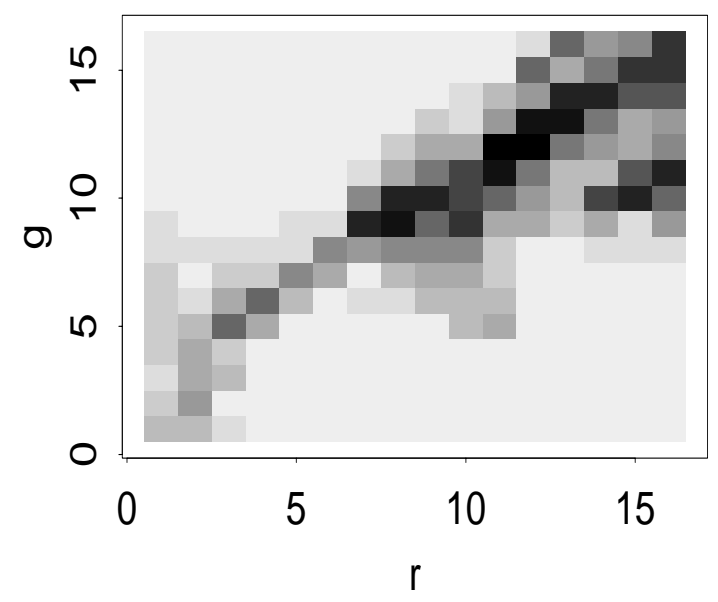

(e)

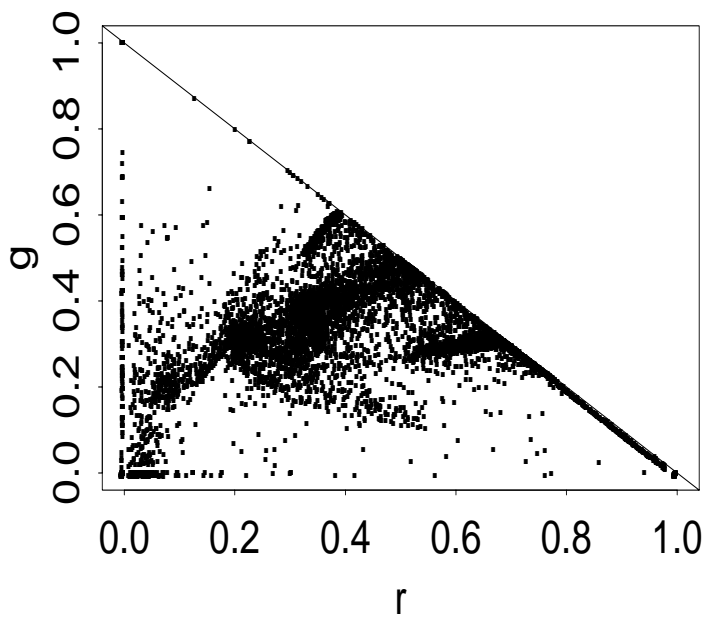

(b)

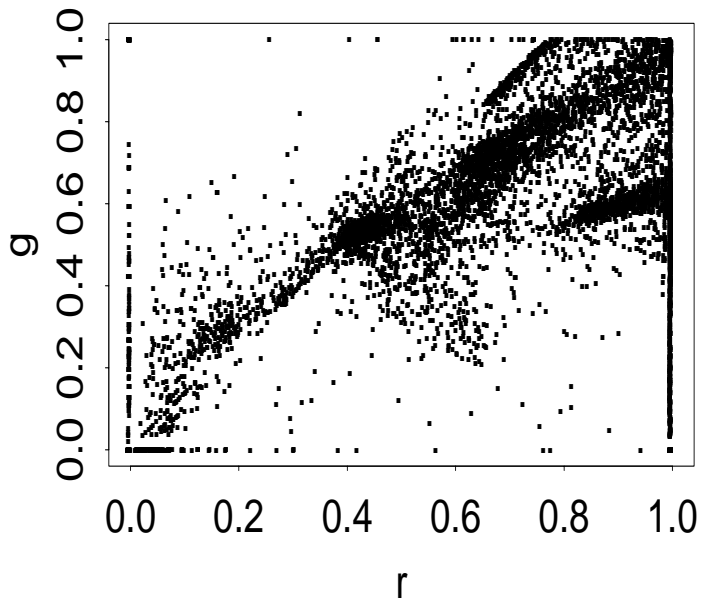

(d)

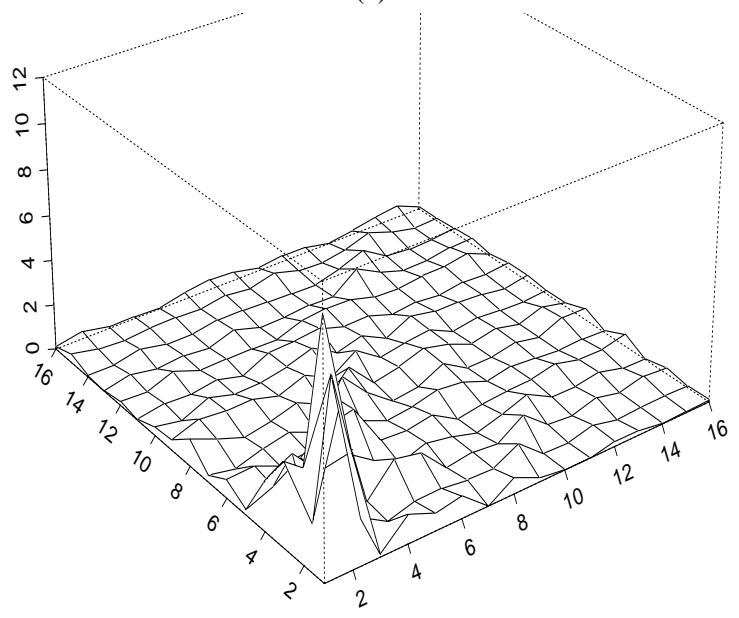

(f)

Figure 5: 


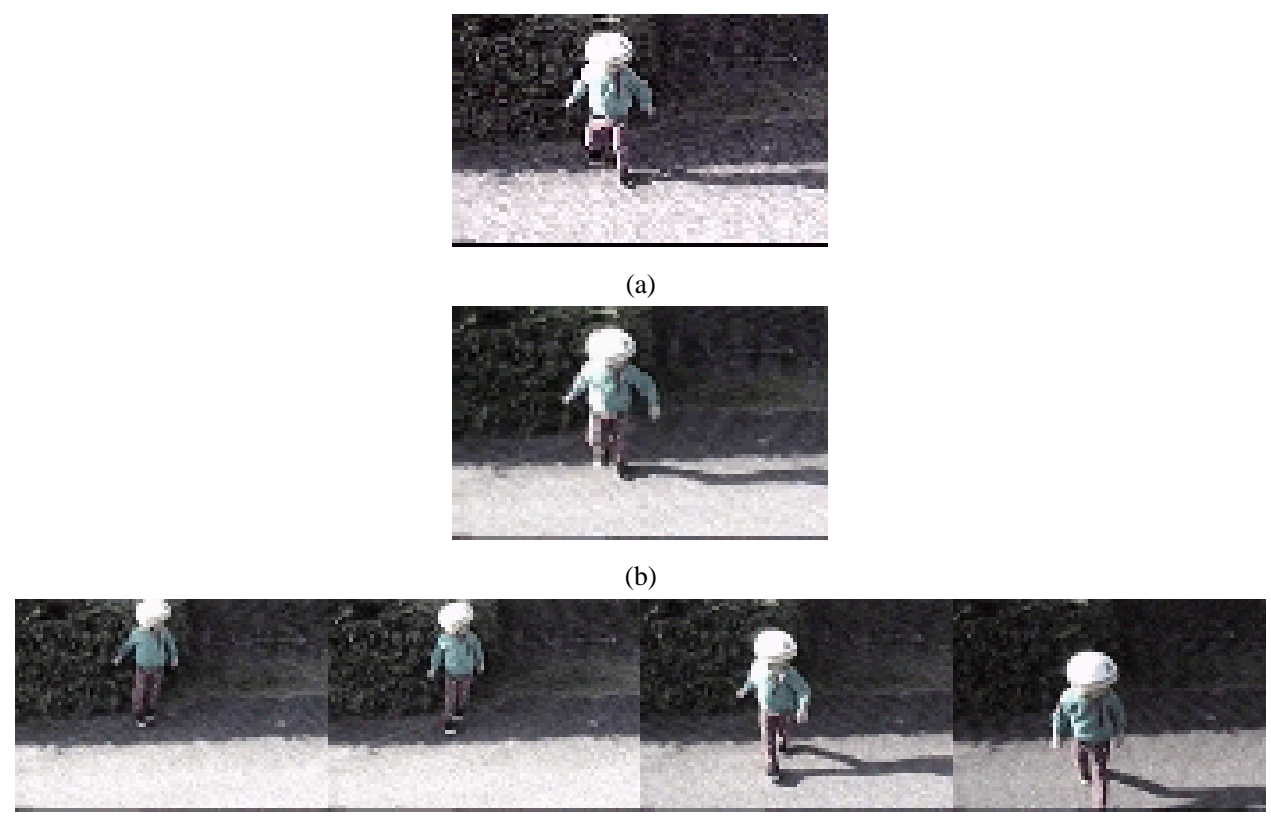

(c)

Figure 6: 


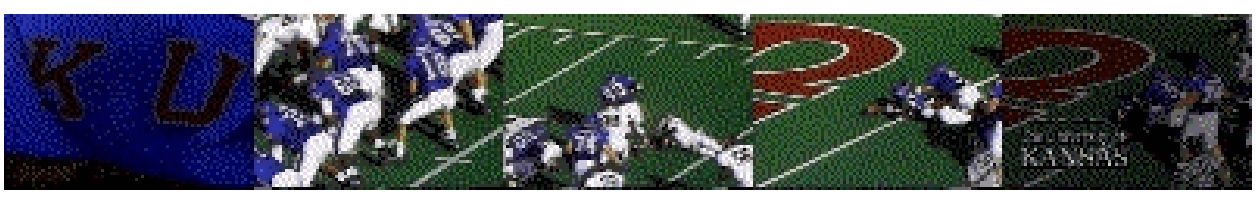

(a)

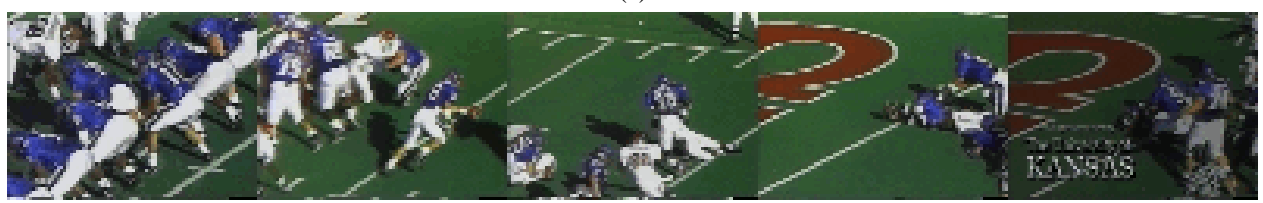

(b)

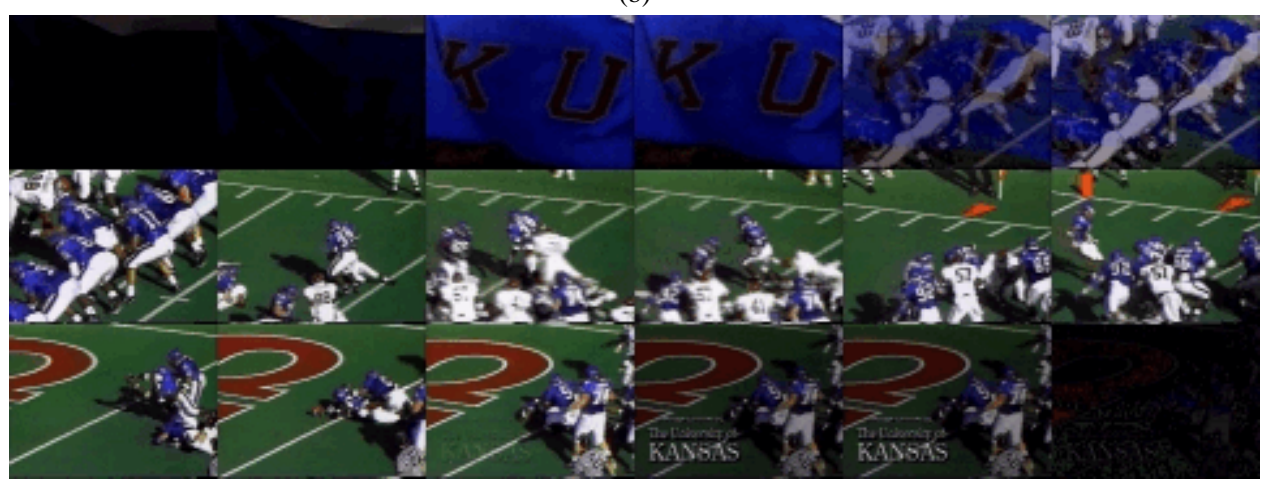

(c)

Figure 7: 


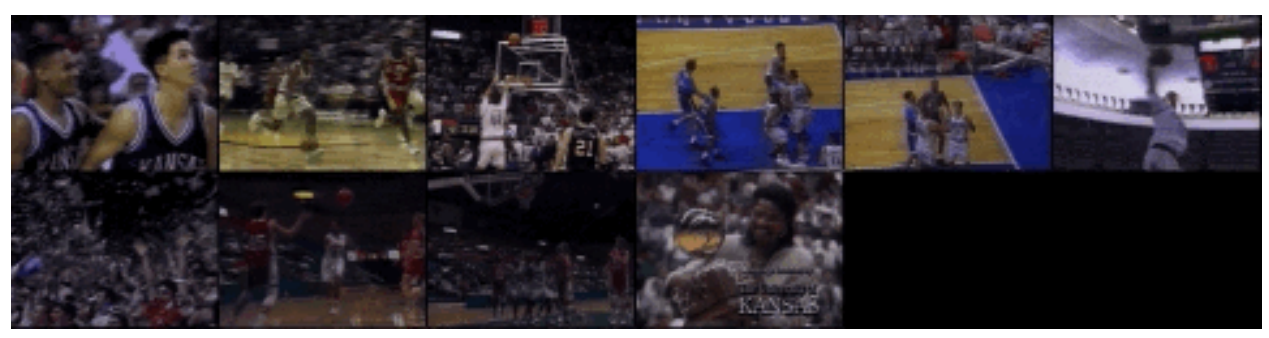

(a)

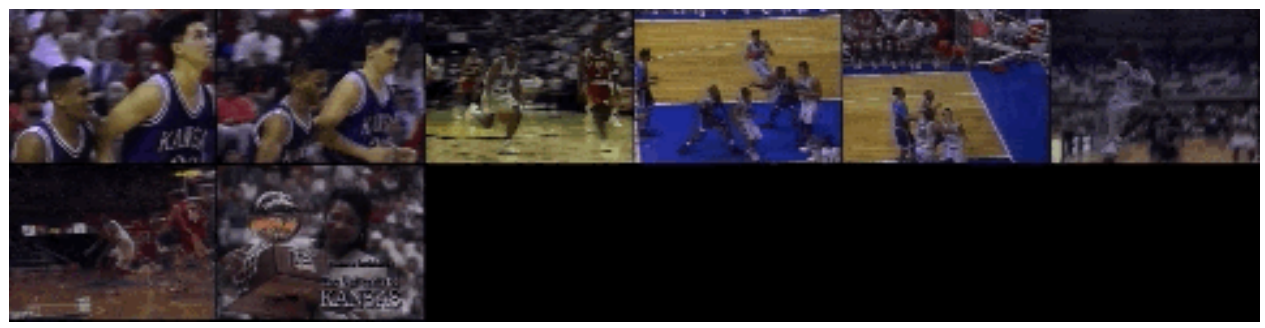

(b)

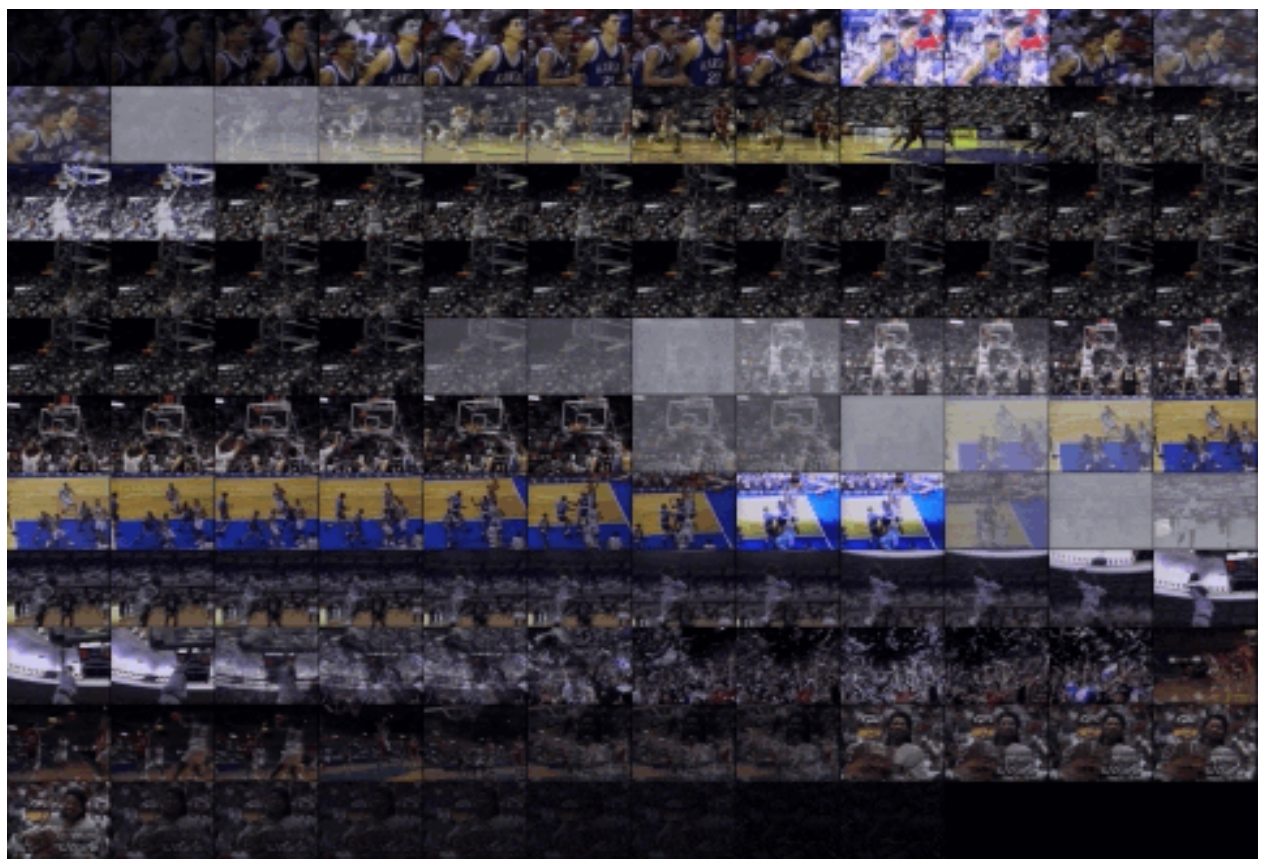

(c)

Figure 8: 